\begin{tabular}{ccc}
\hline & International Journal of Engineering \& Technology, $7(2.12)(2018) 147-150$ \\
SPC & International Journal of Engineering \& Technology \\
Website: $w$ ww.sciencepubco.com/index.php/IJET & Research Paper \\
\hline
\end{tabular}

\title{
A study on the range $\&$ deflection distance observation based on latitude position of artillery gun system
}

\author{
Hwan Il Kang ${ }^{1}$, Gun In Kim ${ }^{2}$, 1Hyun Soo Kim ${ }^{1}$ \\ ${ }^{1}$ Department of Information \& Communications Eng.Myongji University, Yongin, Geonggido, 17058, South Korea \\ ${ }^{2}$ Dept. of Cyber Security, Korea University, Seoul, 34186, South Korea \\ *Corresponding author E-mail:hwanilkang@naver.com
}

\begin{abstract}
Background/Objectives: In this paper, instead of using the six degree of freedom trajectory for a projectile, we consider itsfour degree of freedom trajectory which is the NATO standard trajectory model. We consider the range and deflection distance observation based on latitude position of Artillery Gun System due to Coriolis acceleration.

Methods/Statistical analysis: We obtain the trajectory of a projectile with $155 \mathrm{~mm}$ diameter using the Runge-Kutta method. We obtain the range and deflection distances associated with fire elevation angle when the launch position is located at differentposition of the artillery gun system. Its position is a function of the latitude.

Findings: By changing the latitude of gun position, variation of range and the deflection distances are measured due to Coriolis force. Under the condition that the fire elevation is fixed, the range and deflection distances are calculated by numerical analysis. The maximum of the range is achieved at the equator. The minimum of deflection distance is observed near the Antarctic while the maximum valueof the deflection is observed at the North Pole.

Improvements/Applications: This approach will be a good resource for analysis of delivery accuracy of the artillery gun system based on its position which is a function of latitude.
\end{abstract}

Keywords: Range; Deflection Distance; Coriolis Acceleration; Four Degree of Freedom; Trajectory; Projectile; Latitude Position.

\section{Introduction}

For the anti-aircraft artillery system, the point mass trajectory is used for fire power performance analysis [1], [2]. For the indirect artillery system, the modified point mass trajectory model with four degree freedom or five degree of freedom ${ }^{3}$ may be used. There are two methods of the modified point mass trajectory model with four degree of freedom [4], [5]. The first model is the standard NATO model [4]. The second model is Bradley model [5].Drag force, Magnus force, lift force, gravitational force and Coriolis forces are exerted during the travel of projectiles. The three translational motion equations and one spin rotational force play a major role for thetrajectory equation with four degree of freedom. In this paper, we use the standard NATO trajectory model. By numerical analysis for the standard NATO trajectory model, we calculate the range and deflection distances when the latitude of the launch position of the artillery gun system varies from the South Pole to the North Pole. In Section 2, the standard NATO trajectory model is described. In Section 3, Coriolis acceleration is presented which results in the variation of the range and deflection distances for the artillery gun system. In Section 4, simulation is given and finally conclusions are followed.

\section{The nato standard trajectory model}

The vector of the yaw of repose $\left(\alpha_{e}\right)$, as shown in figure 1, consists of velocity and the acceleration components for the NATO standard form [6-8].

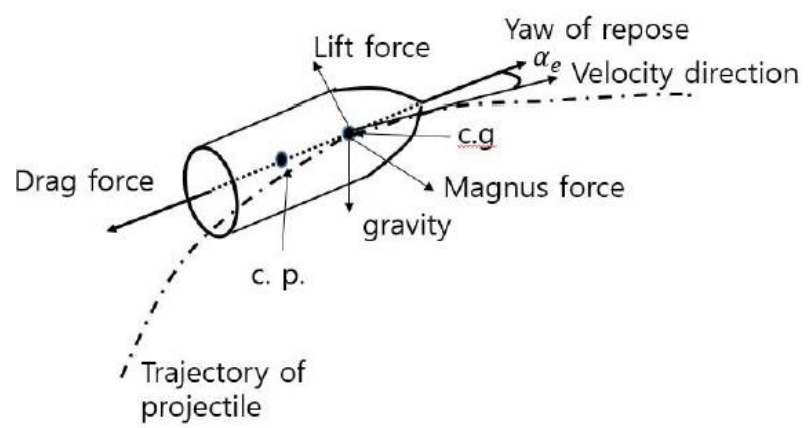

Fig. 1: Yaw of Repose $\left(\alpha_{e}\right)$.

Gravity force $\left(\overrightarrow{F_{g}}\right)$ acts as the magnitude of gravity in the radial direction towards the center of gravity and can be modeled as a function of latitude and azimuth angle. In figure 2 , the $x$ axis is the range direction, $z$ axis is the deflection direction and the $y$ axis is the opposite direction of the gravity direction ${ }^{6}$. lat means the latitude of the launch position and $A Z$ means the azimuth direction at the launch position from North Pole as shown in figure 2. 


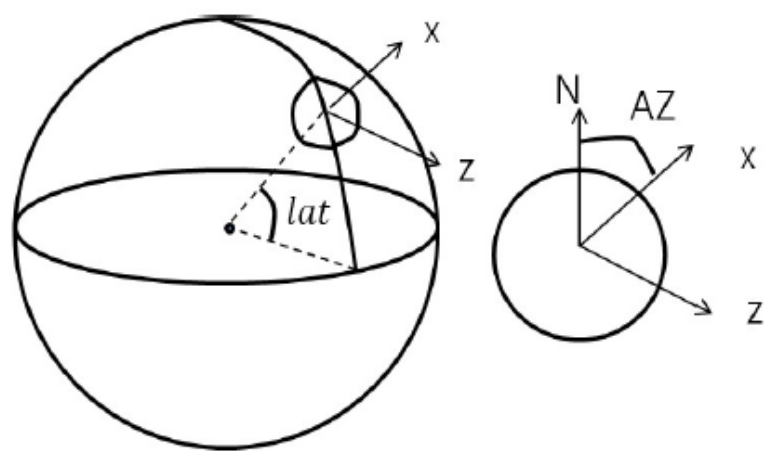

Fig. 2:.Angles Used for Coriolis Acceleration Calculation ${ }^{6}$.

$\overrightarrow{F_{g}}=-m g_{0}($ lat,$A Z)\left[\begin{array}{c}\frac{x}{R_{z}} \\ 1-\frac{2 y}{R_{z}} \\ \frac{z}{R_{z}}\end{array}\right]$

$R_{z}$ means the radius of the earth. In figure 1 , the drag force $\left(\overrightarrow{F_{D}}\right)$ is expressed in equation (2)and its direction is againstthe direction of the direction of the projectile

$$
\overrightarrow{F_{D}}=-S i \frac{\rho}{2}\left[C_{D_{\alpha}}+C_{D_{\alpha^{2}}}\left(Q_{d} \alpha_{e}\right)^{2}\right] v \cdot \vec{v}
$$

where $S$ is the cross section of the projectile, the $\rho$ is the density of air. $C_{D_{\alpha}}, C_{D_{\alpha^{2}}}$ refer to drag coefficient and secondary drag coefficient, respectively.vmeans the speed vector of the projectile. The lift force $\left(\overrightarrow{F_{L}}\right)$ is perpendicular to the direction of the heading perpendicular to the direction of progress, expressed in equation (3).

$$
\overrightarrow{F_{L}}=S f_{L} \frac{\rho}{2}\left[C_{L_{\alpha}}+C_{L_{\alpha^{3}}} \alpha_{e}^{2}\right] v^{2} \overrightarrow{\alpha_{e}}
$$

$C_{L_{\alpha}}$ and $C_{L_{\alpha^{3}}}$ are denoted by the lift coefficient and secondary lift coefficient, respectively. $f_{L}$ represents the lift constant. Magnus force $\left(\overrightarrow{F_{M}}\right)$ can be represented as equation 4 where $Q_{M}$ represents the Magnus constant and $\mathrm{d}$ is the diameter of the bullet, and $C_{M a g-f}$ indicates the Magnus coefficient.

$$
\overrightarrow{F_{M}}=S d Q_{M} \frac{\rho}{2}\left[C_{m a g-f}\right]\left(\overrightarrow{\alpha_{e}} \times \vec{v}\right)
$$

Figure 3 shows the direction of the Magnus force

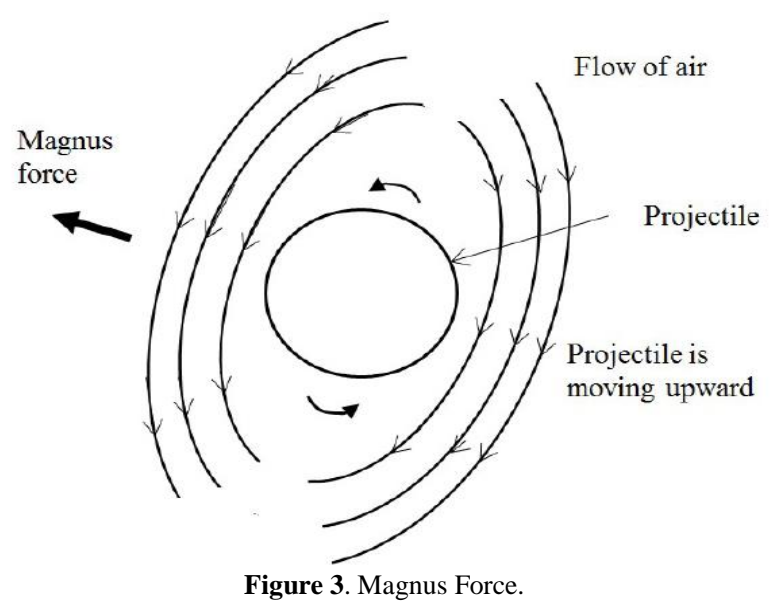

The location of the projectile $(\mathrm{X})$ in the initial position at the fixed coordinate system is expressed as an equation for the $\mathrm{x}, \mathrm{y}$ and $\mathrm{z}$ axes of the mass of the projectile, expressed as follows.

$\mathrm{X}=X_{0}+\int_{0}^{t} u d t$ $\frac{d X_{i}}{d t}=$

The equation of the $\mathrm{x}$-axis is represented as

$\frac{d u_{1}}{d t}=-\frac{\pi \rho i d^{2}}{8 m}\left[C_{D_{\alpha}}+C_{D_{\alpha^{2}}}\left(Q_{d} \alpha_{e}\right)^{2}\right] v v_{1}+\frac{\pi \rho d^{2} f_{L}}{8 m}\left[C_{L_{\alpha}}+\right.$

$\left.C_{L_{\alpha^{3}}} \alpha_{e}^{2}\right] v^{2} \alpha_{e 1}-g_{0} \frac{X_{1}}{R}-\frac{\pi \rho d^{3} Q_{M} p C_{m a g-f}}{8 m}\left(\alpha_{e 2} v_{3}-\alpha_{e 3} v_{2}\right)$

$-2 \Omega\left(-u_{2} \cos (\right.$ lat $) \sin (a z)-u_{3} \sin ($ lat $\left.)\right)$

The equation for the movement of the y-axis is expressible as

$\frac{d u_{2}}{d t}=-\frac{\pi \rho i d^{2}}{8 m}\left[C_{D_{\alpha}}+C_{D_{\alpha^{2}}}\left(Q_{d} \alpha_{e}\right)^{2}\right] v v_{2}+\frac{\pi \rho d^{2} f_{L}}{8 m}\left[C_{L_{\alpha}}+\right.$

$\left.C_{L_{\alpha^{3}}} \alpha_{e}^{2}\right] v^{2} \alpha_{e 2}-g_{0}\left(1-\frac{2 X_{2}}{R}\right)-\frac{\pi \rho d^{3} Q_{M} p C_{m a g-f}}{8 m}\left(\alpha_{e 3} v_{1}-\alpha_{e 1} v_{3}\right)$

$+2 \Omega\left(u_{1} \cos (\right.$ lat $) \sin (a z)+u_{z} \cos ($ lat $\left.) \cos (a z)\right)$

The equation for the movement of the $\mathrm{z}$-axis is expressible as

$\frac{d u_{3}}{d t}=-\frac{\pi \rho i d^{2}}{8 m}\left[C_{D_{\alpha}}+C_{D_{\alpha^{2}}}\left(Q_{d} \alpha_{e}\right)^{2}\right] v v_{3}+\frac{\pi \rho d^{2} f_{L}}{8 m}\left[C_{L_{\alpha}}+\right.$

$\left.C_{L_{\alpha^{3}}} \alpha_{e}^{2}\right] v^{2} \alpha_{e 3}-g_{0} \frac{X_{3}}{R}-\frac{\pi \rho d^{3} Q_{M} p C_{m a g-f}}{8 m}\left(\alpha_{e 1} v_{2}-\alpha_{e 2} v_{1}\right)$

$+2 \Omega\left(u_{1} \sin (\right.$ lat $)-u_{2} \cos ($ lat $\left.) \cos (a z)\right)$

The equation for spin velocity is as follows

$\frac{d p}{d t}=-\frac{\pi \rho d^{4} p v C_{\text {spin }}}{8 I_{x}}$

Here, $I_{x}$ represents the rotational mass of the bullet and the $C_{\text {spin }}$ stands for the spin count. An equation regarding the orientation of the yaw angle $\mathrm{x}, \mathrm{y}$, and $\mathrm{z}$ axis is

$\alpha_{e 1}=-\frac{8 I_{x} p\left(v_{2} \dot{u}_{3}-v_{3} \dot{u}_{2}\right)}{\pi \rho d^{3} C_{M_{\alpha}} v^{4}}$

$\alpha_{e 2}=-\frac{8 I_{x} p\left(v_{3} \dot{u}_{1}-v_{1} \dot{u}_{3}\right)}{\pi \rho d^{3} C_{M_{\alpha}} v^{4}}$

$\alpha_{e 3}=-\frac{8 I_{x} p\left(v_{1} \dot{u}_{2}-v_{2} \dot{u}_{1}\right)}{\pi \rho d^{3} C_{M_{\alpha}} v^{4}}$

The speed at which the calibration point is plotted appears in the following equation (15):

$v_{i}=u_{i}-w_{i}, i=1,2,3$

$v=\sqrt{v_{1}^{2}+v_{2}^{2}+v_{3}^{2}}$

Here, $u_{i}$ and $w_{i}$ represent the speed of the wind and the velocity of wind.

\section{Coriolis acceleration}

Trajectory equations are based on the equation

$m \dot{u}=D F+L F+M F+m g+m \Lambda$

Where $D F, L F, M F, m g$ and $m \Lambda$ mean the drag force, lift force, Magnus force, gravity force and the Coriolis force.

In Figure 1, the vector of yaw of repose is given by

$\alpha_{e}=-\frac{8 I_{\chi} p(v \times \dot{u})}{\pi \rho d^{3}\left(C_{M_{\alpha}}+C_{M_{\alpha^{3}}} \alpha_{e}^{2}\right) v^{4}}$

And the Coriolis acceleration in equation (15) is given by 
$\Lambda_{x}=2 \Omega\left(-u_{y} \cos (L) \sin (A Z)-u_{z} \sin (L)\right)$

$\Lambda_{y}=2 \Omega\left(u_{x} \cos (L) \sin (A Z)+u_{z} \cos (L) \cos (A Z)\right)$

$\Lambda_{z}=2 \Omega\left(u_{x} \sin (L)-u_{y} \cos (L) \cos (A Z)\right)$

The rotational motion of the projectile is given by

$\frac{d p}{d t}=\frac{\pi \rho d^{4} p v C_{\text {spin }}}{8 I_{x}}$ where $I_{x}$ is the projectile's axis moment of inertia, $\mathrm{d}$ is the projectile's diameter, $\Lambda_{x}, \Lambda_{y}, \Lambda_{z}$ are Coriolis's acceleration vector component [6], [7], $\Omega$ is the angular velocity of the Earth around its polar axis and $C_{\text {spin }}$ is the spin damping moment coefficient.

\section{Simulation results}

The characteristic of the projectile and its initial conditions ${ }^{8}$ tabulate in table 1 .

Table 1: Characteristics of Projectile and Related Parameters [8]

\begin{tabular}{|c|c|c|c|c|c|}
\hline $\begin{array}{l}\text { Temperature } \\
\left({ }^{0} \mathrm{C}\right)\end{array}$ & $\begin{array}{l}\text { Projectile } \\
\text { mass }(\mathrm{Kg})\end{array}$ & Projectile diameter(m) & $\begin{array}{l}\text { Spin angular ve- } \\
\text { locity (rad/s) }\end{array}$ & Elevation angle(mil) & $\begin{array}{l}\text { Muzzle veloc- } \\
\text { ity }(\mathrm{m} / \mathrm{s})\end{array}$ \\
\hline 15.0 & 47.5 & 0.155 & 1654.3 & $1000(56.25 \mathrm{deg})$ & $347 \mathrm{~m} / \mathrm{s}$ \\
\hline $\begin{array}{l}\text { Projectile iner- } \\
\text { tia moment }\end{array}$ & Lift factor & QM( Magnus force factor) & $\mathrm{I}$ (form factor) & $\operatorname{Gravity}\left(\mathrm{m} / \mathrm{s}^{2}\right)$ & $\mathrm{Q}_{\mathrm{d}}$ (Yaw drag factor) \\
\hline 0.16 & 1 & 1 & 1 & 9.8005 & 1.2 \\
\hline
\end{tabular}

Table 2: Various Aerodynamic Coefficients [8]

\begin{tabular}{|c|c|c|c|c|c|c|c|}
\hline$M$ & $C_{D_{\alpha}}$ & $C_{D_{\alpha^{2}}}$ & $C_{L_{\alpha}}$ & $C_{L_{\alpha^{4}}}$ & $C_{m a g-f}$ & $C_{M_{\alpha}}$ & $C_{\text {spin }}$ \\
\hline 0.40 & 0.138 & 4.1710 & 1.302 & 20 & -0.510 & 3.725 & -0.01320 \\
\hline 0.60 & 0.138 & 4.1710 & 1.302 & 20 & -0.510 & 3.733 & -0.01320 \\
\hline 0.70 & 0.139 & 4.4305 & 1.311 & 20 & -0.510 & 3.849 & -0.01280 \\
\hline 0.75 & 0.140 & 4.5500 & 1.430 & 20 & -0.510 & 4.220 & -0.01260 \\
\hline 0.80 & 0.141 & 4.6895 & 1.439 & 20 & -0.510 & 4.276 & -0.01240 \\
\hline 0.85 & 0.148 & 4.9560 & 1.452 & 20 & -0.545 & 4.553 & -0.01210 \\
\hline 0.875 & 0.152 & 5.0840 & 1.458 & 20 & -0.560 & 4.692 & -0.01195 \\
\hline 0.90 & 0.156 & 5.2320 & 1.474 & 20 & -0.575 & 4.830 & -0.01180 \\
\hline 0.925 & 0.177 & 5.4915 & 1.423 & 20 & -0.650 & 4.686 & -0.01170 \\
\hline 0.950 & 0.199 & 5.7505 & 1.371 & 20 & -0.725 & 4.542 & -0.01155 \\
\hline 0.975 & 0.244 & 6.0280 & 1.436 & 20 & -0.695 & 4.565 & -0.01155 \\
\hline 1.0 & 0.290 & 6.2950 & 1.490 & 20 & -0.665 & 4.587 & -0.01150 \\
\hline 1.025 & 0.309 & 6.5755 & 1.551 & 20 & -0.635 & 4.522 & -0.01160 \\
\hline 1.050 & 0.329 & 6.8555 & 1.621 & 20 & -0.605 & 4.457 & -0.01175 \\
\hline 1.10 & 0.326 & 7.4470 & 1.694 & 20 & -0.575 & 4.516 & -0.01150 \\
\hline 1.20 & 0.318 & 8.0510 & 1.802 & 20 & -0.510 & 4.572 & -0.01150 \\
\hline 1.35 & 0.305 & 7.6075 & 1.945 & 20 & -0.510 & 4.599 & -0.01130 \\
\hline 1.50 & 0.291 & 7.1545 & 2.089 & 20 & -0.510 & 4.708 & -0.01110 \\
\hline 1.75 & 0.269 & 6.7155 & 2.251 & 20 & -0.510 & 4.723 & -0.01100 \\
\hline 2.00 & 0.249 & 6.2655 & 2.411 & 20 & -0.510 & 4.649 & -0.01110 \\
\hline 2.25 & 0.233 & 6.0135 & 2.517 & 20 & -0.510 & 4.613 & -0.01105 \\
\hline 2.5 & 0.216 & 5.7620 & 2.614 & 20 & -0.510 & 4.573 & -0.01100 \\
\hline 3.0 & 0.194 & 5.2330 & 2.576 & 20 & -0.510 & 4.289 & -0.01070 \\
\hline
\end{tabular}

Table 2 tabulates the aerodynamic coefficients [8] used for ballistic equations.

Assume that the fire elevation angle is $1000 \mathrm{mil}$. We obtain the trajectory of a projectile with $155 \mathrm{~mm}$ diameter using the Runge-Kutta method. Under this condition, we obtain the range and deflection distance for every latitude from the South Pole to the North Pole. As shown in figure 4, the maximum value of the range is given at the equator. In figure 5, the minimum value of deflection distance is given near the latitudes of 70 degree in the southern hemisphere and the maximum value of the deflection distance is achieved at the North Pole.

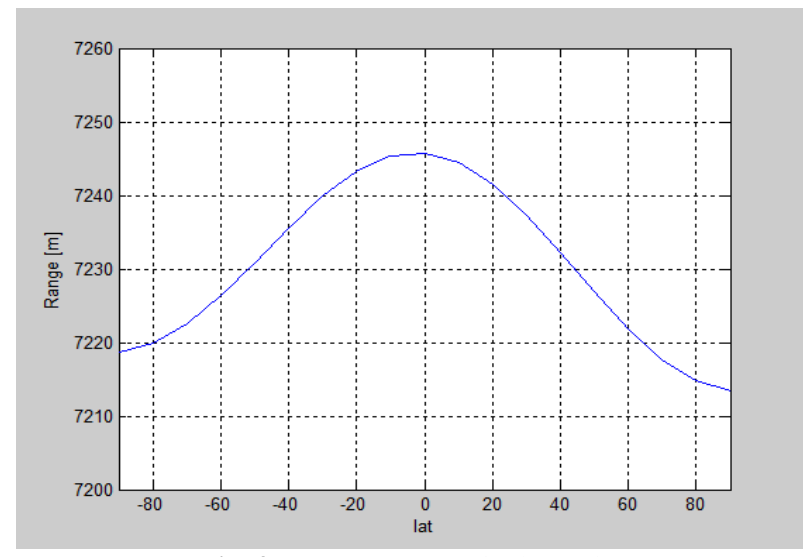

Fig. 4:.Range Versus Latitude Angle.

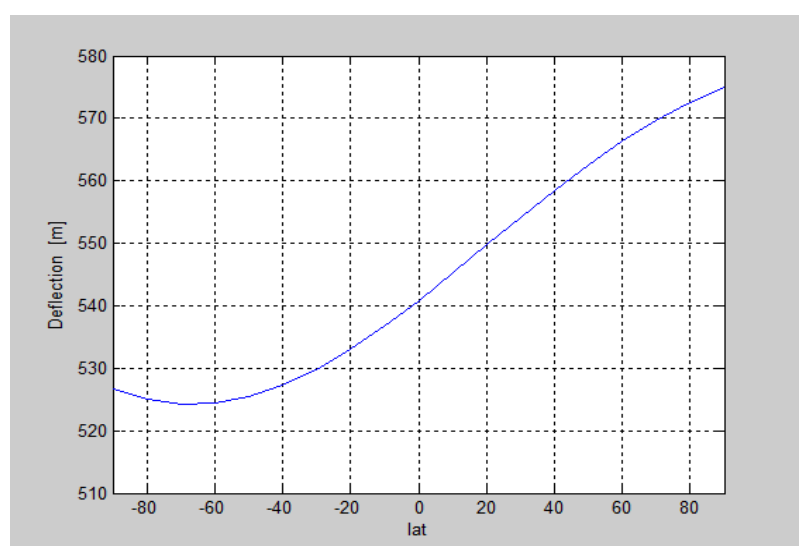

Fig. 5: Deflection Distance versus Latitude Angle.

\section{Conclusions}

For the modified point mass trajectory model, the Coriolis acceleration vector results in variation of range and deflection distance. We observe the relationship between the latitude and distances such as range and deflection distance. When the latitude varies, the variations of range and deflection distance are measured. This result will be useful resources for analysis of fire power performance of the indirect artillery system [9], [10]. 


\section{Acknowledgment}

This work was supported by the Research program (the specialized research center for future ground system analysis (Contract No. 311165-911074201) funded by the agency for defense development in the republic of Korea.

\section{References}

[1] Kang H. I., PyunJ. J., Kim H. S., A Study on Algebraic Solutions of Trajectory Equations, Indian Journal of Science \& Technology 8(S1), 2014, pp.206-209.

[2] Kang, H. I.,Pyun J. J.,Kim, H. S., A Study on Prediction of Probability of Hit for an Anti-aircraft Artillery, Indian Journal of Science \& Technology 9(S1), 2016, pp.1-5.

[3] Morento J. A., the Modified Point Mass and Five Degrees of Freedom Trajectory Models, Stanag 4355 (Edition 3), North Atlantic Treaty Organization (NATO), NATO Standardization Agency (NSA), 2009.

[4] Baranowski, L.,"Feasibility Analysis of the Modified Point Mass Trajectory Model for the Need of Ground Artillery Fire Control Systems," Journal of Theoretical \& Applied Mechanics, 51, (3), 2013, pp. 511 522.

[5] Barbosa L. M., Blanco A. L. M., Dutra L. M., Santana D. P., Abrunhosa J. D. M., A Critical Evaluation of Three Models of External Ballistics, $18^{\text {th }}$ International Congress of Mechanical Engineering, November 6-11, OuroPreto, MG. 2005.

[6] Carlucci Donald E.,Jacobson Sidney S., Ballistics: Theory \& Design of Gun \& Ammunition, CRC Press, Taylor \& Francis Group, Boca Raton, FL, USA, 2014, p. 265.

[7] McCoy, Robert L. Modern Exterior Ballistics, TheLaunchand Fligh Dynamics of Symmetrical Projectiles, Schiffer Military History Publishing Limited, 2012, p 191.

[8] Rastislav B., KomendaJan, "Analysis of $155 \mathrm{mmERFB} / \mathrm{BB}$ Projectile Trajectory", Advances in MT, 2006, pp.91-114.

[9] Driels, Morris. Weaponeering: Conventional weapon system effectiveness, AIAA Educational Series, AIAA Inc, Reston, VA, 2013, pp. 134-156.

[10] Lim Wee Yeow, Predicting the accuracy of unguided artillery projectiles, MS thesis, Naval Postgraduate School, Advisor: Morris R. Driels, 2016.

[11] Lee JR, Kim SA, Yoo JW \& Kang YK (2007), The present status of diabetes education and the role recognition as a diabetes educator of nurses in korea. Diabetes Research and Clinical Practice 77, 199204.

[12] McMahon GT, Gomes HE, Hohne SH, Hu TM, Levine BA \& Conlin PR (2005), Web-based care management in patients with poorly controlled diabetes. Diabetes Care 28, 1624-1629.

[13] Thakurdesai PA, Kole PL \& Pareek RP (2004), Evaluation of the quality and contents of diabetes mellitus patient education on Internet. Patient Education and Counseling 53, 309-313. 